IJMMS 25:4 (2001) 231-237

PII. S0161171201004537

http://ijmms.hindawi.com

(C) Hindawi Publishing Corp.

\title{
A CHANGE OF SCALE FORMULA FOR WIENER INTEGRALS OF CYLINDER FUNCTIONS ON THE ABSTRACT WIENER SPACE II
}

\author{
YOUNG SIK KIM
}

(Received 24 January 2000)

\begin{abstract}
We show that for certain bounded cylinder functions of the form $F(x)=$ $\hat{\mu}\left(\left(h_{1}, x\right)^{\sim}, \ldots,\left(h_{n}, x\right)^{\sim}\right), x \in B$, where $\hat{\mu}: \mathbb{R}^{n} \rightarrow \mathbb{C}$ is the Fourier-transform of the complexvalued Borel measure $\mu$ on $\mathscr{B}\left(\mathbb{R}^{n}\right)$, the Borel $\sigma$-algebra of $\mathbb{R}^{n}$ with $\|\mu\|<\infty$, the analytic Feynman integral of $F$ exists, although the analytic Feynman integral, $\lim _{z \rightarrow-i q} I^{\text {aw }}(F ; z)=$ $\lim _{z \rightarrow-i q}(z / 2 \pi)^{n / 2} \int_{\mathbb{R}^{n}} f(\vec{u}) \exp \left\{-(z / 2)|\vec{u}|^{2}\right\} d \vec{u}$, do not always exist for bounded cylinder functions $F(x)=f\left(\left(h_{1}, x\right)^{\sim}, \ldots,\left(h_{n}, x\right)^{\sim}\right), x \in B$. We prove a change of scale formula for Wiener integrals of $F$ on the abstract Wiener space.
\end{abstract}

2000 Mathematics Subject Classification. Primary 28C20.

1. Introduction. In [3], Kim showed that for $F \in \mathscr{F}(n ; p), 1 \leq p \leq \infty$, the analytic Wiener integral exists and for $F \in \mathscr{F}(n ; 1)$, the analytic Feynman integral exists and can be expressed as the limit of Wiener integrals and later he proved the change of scale formula for Wiener integrals for $F \in \mathscr{F}(n ; p), 1 \leq p \leq \infty$, where for $1 \leq p<\infty, \mathscr{F}(n ; p)$ is the class of cylinder functions $F$ of the form $F(x)=$ $f\left(\left(h_{1}, x\right)^{\sim}, \ldots,\left(h_{n}, x\right)^{\sim}\right)$ and $f: \mathbb{R}^{n} \rightarrow \mathbb{R}$ is in $L_{p}\left(\mathbb{R}^{n}\right)$, and $\mathscr{F}(n ; \infty)$ is the class of such cylinder functions $F$, where $f: \mathbb{R}^{n} \rightarrow \mathbb{R}$ is in $C_{0}\left(\mathbb{R}^{n}\right)$, the space of bounded continuous functions on $\mathbb{R}^{n}$ that vanish at infinity. But for $1<p \leq \infty$, the analytic Feynman integral of $F \in \mathscr{F}(n ; p)$ do not always exist even if $F(x)=f\left(\left(h_{1}, x\right)^{\sim}, \ldots,\left(h_{n}, x\right)^{\sim}\right)$ is a bounded cylinder function, as we cannot apply the Lebesgue dominated convergence theorem to the limit whenever $z \rightarrow-i q$ through $\mathbb{C}_{+} ; \lim _{z \rightarrow-i q} I^{\mathrm{aw}}(F ; z)=$ $\lim _{z \rightarrow-i q}(z / 2 \pi)^{n / 2} \int_{\mathbb{R}^{n}} f(\vec{u}) \exp \left\{-(z / 2)|\vec{u}|^{2}\right\} d \vec{u}$.

In this paper, we show that the analytic Feynman integral of $F$ exists for certain bounded cylinder functions of the form $F(x)=\hat{\mu}\left(\left(h_{1}, x\right)^{\sim}, \ldots,\left(h_{n}, x\right)^{\sim}\right), x \in B$, where $\hat{\mu}: \mathbb{R}^{n} \rightarrow \mathbb{C}$ is the Fourier-transform of the complex-valued Borel measure $\mu$ on $\mathscr{B}\left(\mathbb{R}^{n}\right)$, the Borel $\sigma$-algebra of $\mathbb{R}^{n}$ with $\|\mu\|<\infty$. We establish the relationships between analytic Wiener integrals, and analytic Feynman integrals, and we show that the analytic Feynman integral of $F$ can be expressed as the limit of a sequence of Wiener integrals. Later, we prove a change of scale formula for Wiener integrals of $F$ on the abstract Wiener space.

2. Definitions. Let $H$ be a real separable infinite-dimensional Hilbert space with inner product $\langle\cdot, \cdot\rangle$ and norm $\|\cdot\|=\sqrt{\langle\cdot, \cdot\rangle}$. Let $\|\cdot\|_{0}$ be a measurable norm on $H$ with respect to the Gauss measure $\mu$. Let $B$ denote the completion of $H$ with respect to $\|\cdot\|_{0}$. Let $i$ denote the natural injection from $H$ into $B$. The adjoint operator $i^{*}$ of $i$ is 
one-to-one and maps $B^{*}$ continuously onto a dense subset of $H^{*}$, where $H^{*}$ and $B^{*}$ are topological duals of $H$ and $B$, respectively. By identifying $H$ with $H^{*}$ and $B^{*}$ with $i^{*} B^{*}$, we have a triplet $\left(B^{*}, H, B\right)$ such that $B^{*} \subset H^{*} \equiv H \subset B$ and $\langle h, x\rangle=(h, x)$ for all $h$ in $B^{*}$ and $x$ in $H$, where $(\cdot, \cdot)$ denotes the natural dual pairing between $B^{*}$ and $B$. By a well-known result of Gross [3], $\mu \cdot i^{-1}$ has a unique countably additive extension $m$ to the Borel $\sigma$-algebra $\mathscr{B}(B)$ on $B$. The triplet $(B, H, m)$ is called an abstract Wiener space and $m$ is called a Wiener measure. We denote the Wiener integral of a functional $F$ by $\int_{B} F(x) d m(x)$. For more details see [1, 3].

Let $\left\{e_{j}\right\}_{j=1}^{\infty}$ denote a complete orthonormal system in $H$ such that $e_{j}$ 's are in $B^{*}$. For each $h \in H$ and $x \in B$, we define a stochastic inner product $(\cdot, \cdot)^{\sim}$ between $H$ and $B$ as follows:

$$
(h, x)^{\sim}= \begin{cases}\lim _{n \rightarrow \infty} \sum_{j=1}^{n}\left\langle h, e_{j}\right\rangle\left(e_{j}, x\right), & \text { if the limit exists, } \\ 0, & \text { otherwise. }\end{cases}
$$

It is well known [2] that for every $h \in H,(h, x)^{\sim}$ exists for $m$-a.e. $x$ in $B$ and it has a Gaussian distribution with mean zero and variance $|h|^{2}$. Furthermore, it is easy to show that $(h, x)^{\sim}=(h, x)$ for $m$-a.e. $x$ in $B$ if $h \in B^{*},(h, x)^{\sim}$ is essentially independent of the complete orthonormal set used in its definition, and finally we show that if $\left\{h_{1}, \ldots, h_{k}\right\}$ is an orthonormal set of elements in $H$, then $\left(h_{1}, x\right)^{\sim}, \ldots,\left(h_{k}, x\right)^{\sim}$ are independent Gaussian functionals with mean zero and variance one. Note that if both $h$ and $x$ are in $H$, then $(h, x)^{\sim}=\langle h, x\rangle$.

Throughout this paper, let $\mathbb{R}^{n}$ denote the $n$-dimensional Euclidean space and let $\mathbb{C}, \mathbb{C}_{+}$, and $\mathbb{C}_{+}^{\sim}$ denote the complex numbers, the complex numbers with positive real part, and the nonzero complex numbers with nonnegative real part, respectively.

DEFINITION 2.1. Let $(B, H, m)$ be an abstract Wiener space. A function $F$ is called a cylinder function on $B$ if there exists a finite subset $\left\{g_{1}, \ldots, g_{k}\right\}$ of $H$ such that

$$
F(x)=\psi\left(\left(g_{1}, x\right)^{\sim}, \ldots,\left(g_{k}, x\right)^{\sim}\right), \quad x \in B,
$$

where $\psi$ is a complex-valued Borel measurable function on $\mathbb{R}^{k}$. It is easy to show that there exists a linearly independent set $\left\{h_{1}, \ldots, h_{n}\right\}$ of $H$ such that every cylinder function $F$ of the form (2.2) is expressed as

$$
F(x)=f\left(\left(h_{1}, x\right)^{\sim}, \ldots,\left(h_{n}, x\right)^{\sim}\right), \quad x \in B,
$$

where $f$ is a complex-valued Borel measurable function on $\mathbb{R}^{n}$. Thus we lose no generality in assuming that every cylinder function on $B$ is of the form (2.3).

DEFINITION 2.2. Let $F$ be a complex-valued measurable function on $B$ such that the integral

$$
J(F ; \lambda)=\int_{B} F\left(\lambda^{-1 / 2} x\right) d m(x)
$$

exists for all real $\lambda>0$. If there exists a function $J^{*}(F ; z)$ analytic on $\mathbb{C}_{+}$such that 
$J^{*}(F ; \lambda)=J(F ; \lambda)$ for all real $\lambda>0$, then we define $J^{*}(F ; z)$ to be the analytic Wiener integral of $F$ over $B$ with parameter $z$, and for each $z \in \mathbb{C}_{+}$, we write

$$
I^{\mathrm{aw}}(F ; z)=J^{*}(F ; z) .
$$

Let $q$ be a nonzero real number and let $F$ be a function on $B$ whose analytic Wiener integral exists for each $z$ in $\mathbb{C}_{+}$. If the following limit exists, then we call it the analytic Feynman integral of $F$ over $B$ with parameter $q$, and we write

$$
I^{\mathrm{af}}(F ; q)=\lim _{z \rightarrow-i q} I^{\mathrm{aw}}(F ; z),
$$

where $z$ approaches $-i q$ through $\mathbb{C}_{+}$and $i^{2}=-1$.

DEFINITION 2.3. Let $M\left(\mathbb{R}^{n}\right)$ denote the space of complex-valued Borel measures on $\mathscr{B}\left(\mathbb{R}^{n}\right)$, the Borel $\sigma$-algebra of $\mathbb{R}^{n}$. It is well known that a complex-valued Borel measure $\mu$ necessarily has a finite total variation $\|\mu\|$, and $\mu\left(\mathbb{R}^{n}\right)$ is a Banach algebra under the norm $\|\cdot\|$ and with convolution as multiplication.

Let $\mu$ be in $\mu\left(\mathbb{R}^{n}\right)$. Then the Fourier transformation $\hat{\mu}$ of $\mu$ is a complex-valued function defined on $\mathbb{R}^{n}$ by the formula

$$
\hat{\mu}(\vec{u})=\int_{\mathbb{R}^{n}} \exp \{i\langle\vec{u}, \vec{v}\rangle\} \mu(d \vec{v}), \quad \vec{u} \in \mathbb{R}^{n},
$$

where $\vec{u}=\left(u_{1}, \ldots, u_{n}\right)$ and $\vec{v}=\left(v_{1}, \ldots, v_{n}\right)$ are in $\mathbb{R}^{n}$, and $\langle\vec{u}, \vec{v}\rangle=\sum_{j=1}^{n} u_{j} v_{j}$.

We will close this section by mentioning the following useful theorem which is called the Wiener integration formula.

THEOREM 2.4. Let $(B, H, m)$ be an abstract Wiener space and let $\left\{h_{1}, \ldots, h_{n}\right\}$ be an orthonormal set of elements in $H$. Let $F: B \rightarrow \mathbb{C}$ be a function defined by the formula

$$
F(x)=f\left(\left(h_{1}, x\right)^{\sim}, \ldots,\left(h_{n}, x\right)^{\sim}\right), \quad x \in B,
$$

where $f: \mathbb{R}^{n} \rightarrow \mathbb{C}$ is a Lebesgue measurable function. Then

$$
\int_{B} f\left(\left(h_{1}, x\right)^{\sim}, \ldots,\left(h_{n}, x\right)^{\sim}\right) d m(x)=\left(\frac{1}{2 \pi}\right)^{n / 2} \int_{\mathbb{R}^{n}} f(\vec{u}) \exp \left\{-\frac{1}{2}|\vec{u}|^{2}\right\} d \vec{u},
$$

where $\vec{u}=\left(u_{1}, \ldots, u_{n}\right) \in \mathbb{R}^{n},|\vec{u}|^{2}=\sum_{j=1}^{n} u_{j}^{2}$, and $d \vec{u}=d u_{1} \cdots d u_{n}$.

In the next section, we use several times the following well-known integration formula

$$
\int_{\mathbb{R}} \exp \left\{-a u^{2}+i b u\right\} d u=\sqrt{\frac{\pi}{a}} \exp \left\{-\frac{b^{2}}{4 a}\right\},
$$

where $a$ is a complex number with $\operatorname{Re} a>0, b$ is a real number, and $i^{2}=-1$.

3. The main results. In this paper, we give a class of a certain bounded cylinder functions of the form $F(x)=f\left(\left(h_{1}, x\right)^{\sim}, \ldots,\left(h_{n}, x\right)^{\sim}\right), x \in B$, such that $f: \mathbb{R}^{n} \rightarrow \mathbb{C}$ 
is bounded, whose analytic Wiener and analytic Feynman integral of $F$ exist and we show that the analytic Feynman integral of such cylinder functions can be expressed as the limit of Wiener integrals. Later, we prove a change of scale formula for Wiener integrals of such cylinder functions on the abstract Wiener space.

Define the function $F: B \rightarrow \mathbb{C}$ by

$$
F(x)=\hat{\mu}\left(\left(h_{1}, x\right)^{\sim}, \ldots,\left(h_{n}, x\right)^{\sim}\right), \quad x \in B,
$$

where $\hat{\mu}$ is the Fourier transform of complex-valued Borel measures $\mu$ in $\mu\left(\mathbb{R}^{n}\right)$ and $\mathcal{M}\left(\mathbb{R}^{n}\right)$ is as in Definition 2.3. Then $F: B \rightarrow \mathbb{C}$ is a bounded cylinder function, as $|\hat{\mu}(\vec{u})| \leq\|\mu\|<\infty$.

First, we show that the analytic Wiener and the analytic Feynman integrals of the function $F$ exist.

THEOREM 3.1. Let $(B, H, m)$ be an abstract Wiener space and let $\left\{h_{1}, \ldots, h_{n}\right\}$ be an orthonormal set of elements in $H$. Let $F: B \rightarrow \mathbb{C}$ be given by (3.1). Then the analytic Wiener and the analytic Feynman integrals of $F$ exist, and for every $z \in \mathbb{C}_{+}$,

$$
I^{\mathrm{aw}}(F ; z)=\int_{\mathbb{R}^{n}} \exp \left\{-\frac{1}{2 z}|\vec{v}|^{2}\right\} \mu(d \vec{v})
$$

and for every nonzero real number $q$,

$$
I^{\mathrm{af}}(F ; q)=\int_{\mathbb{R}^{n}} \exp \left\{-\frac{i}{2 q}|\vec{v}|^{2}\right\} \mu(d \vec{v}),
$$

where $\vec{v}=\left(v_{1}, \ldots, v_{n}\right) \in \mathbb{R}^{n}$ and $|\vec{v}|^{2}=\sum_{j=1}^{n} v_{j}^{2}$.

Proof. By Fubini's theorem and by Theorem 2.4 and by (2.10), we have that for all positive real number $\lambda$,

$$
\begin{aligned}
J(F: \lambda) & \equiv \int_{B} F\left(\lambda^{-1 / 2} x\right) d m(x)=\int_{B} \hat{\mu}\left(\left(h_{1}, \lambda^{-1 / 2} x\right)^{\sim}, \ldots,\left(h_{n}, \lambda^{-1 / 2} x\right)^{\sim}\right) d m(x) \\
& =\int_{\mathbb{R}^{n}} \int_{B} \exp \left[i \lambda^{-1 / 2} \sum_{j=1}^{n} v_{j}\left(h_{j}, x\right)^{\sim}\right] d m(x) d \mu(\vec{v}) \\
& =\left(\frac{1}{2 \pi}\right)^{n / 2} \int_{\mathbb{R}^{n}} \int_{\mathbb{R}^{n}} \exp \left[i \lambda^{-1 / 2} \sum_{j=1}^{n} v_{j} u_{j}\right] \exp \left[-\frac{1}{2} \sum_{j=1}^{n} u_{j}^{2}\right] d u_{1} \cdots d u_{n} d \mu(\vec{v}) \\
& =\int_{\mathbb{R}^{n}} \exp \left[-\frac{1}{2 \lambda} \sum_{j=1}^{n} v_{j}^{2}\right] d \mu(\vec{v}) .
\end{aligned}
$$

Now let $J^{*}(F: z)=\int_{\mathbb{R}^{n}} \exp \left\{-(1 / 2 z) \sum_{j=1}^{n} v_{j}^{2}\right\} d \mu(\vec{v}), z \in \mathbb{C}_{+}$. Then $J^{*}(F: \lambda)=$ $J(F: \lambda)$ for all real $\lambda>0$. By dominated convergence theorem, $J^{*}(F: z)$ is continuous on $\mathbb{C}_{+}$. Since $\exp \left\{-(1 / 2 z) \sum_{j=1}^{n} v_{j}^{2}\right\}$ is analytic on $\mathbb{C}_{+}$for each $\vec{v}=\left(v_{1}, \ldots, v_{n}\right) \in \mathbb{R}^{n}$, we have that $\int_{\Gamma} \exp \left\{-(1 / 2 z) \sum_{j=1}^{n} v_{j}^{2}\right\} d z=0$ for all rectifiable simple closed curve $\Gamma$ lying $\mathbb{C}_{+}$by Cauchy integral theorem. As $\left|\exp \left\{-(1 / 2 z) \sum_{j=1}^{n} v_{j}^{2}\right\}\right| \leqslant 1$ for all $z \in$ $\mathbb{C}_{+}$, we can apply Fubini's theorem to the integral $\int_{\Gamma} J^{*}(F: z) d z$ and then we have $\int_{\Gamma} J^{*}(F: z) d z=0$. By Morera's theorem, $J^{*}(F: z)$ is an analytic function of $z$ in $\mathbb{C}_{+}$. 
Therefore the analytic Wiener integral $I^{\text {aw }}(F: z)$ exist and we have (3.2). To prove (3.3), let $f_{n}(\vec{v})=\exp \left\{-\left(1 / z_{n}\right)|\vec{v}|^{2}\right\}, z_{n} \in \mathbb{C}^{+}$and let $z_{n} \rightarrow-i q$ whenever $n \rightarrow \infty$. Then $f_{n}(\vec{v}) \rightarrow f(\vec{v}) \equiv \exp -\left\{(i / 2 q) \vec{v}^{2}\right\}$, whenever $z_{n} \rightarrow-i q$ and $\left|f_{n}(\vec{v})\right| \leq 1$, for all $z_{n} \in \mathbb{C}^{+}$. By the bounded convergence theorem, we have (3.3), as $\left\|\mu\left(\mathbb{R}^{n}\right)\right\|<\infty$.

In order to obtain our main results, we need the following lemma.

LEMMA 3.2. Let $(B, H, m)$ be an abstract Wiener space and let $\left\{h_{1}, \ldots, h_{n}\right\}$ be an orthonormal set of elements in $H$. Let $F: B \rightarrow \mathbb{C}$ be given by (3.1). Then for every $z \in \mathbb{C}_{+}$, the functional

$$
\exp \left\{\frac{1-z}{2} \sum_{j=1}^{n}\left[\left(h_{j}, x\right)^{\sim}\right]^{2}\right\} F(x)
$$

is Wiener integrable on $B$.

Proof. By Theorem 2.4, we have that for every $z \in \mathbb{C}_{+}$,

$$
\int_{B} \exp \left\{\frac{1-z}{2} \sum_{j=1}^{n}\left[\left(h_{j}, x\right)^{\sim}\right]^{2}\right\} F(x) d m(x)=\left(\frac{1}{2 \pi}\right)^{n / 2} \int_{\mathbb{R}^{n}} \hat{\mu}(\vec{u}) \exp \left\{-\frac{z}{2}|\vec{u}|^{2}\right\} d \vec{u},
$$

where $\vec{u}=\left(u_{1}, \ldots, u_{n}\right) \in \mathbb{R}^{n},|\vec{u}|^{2}=\sum_{j=1}^{n} u_{j}^{2}$, and $d \vec{u}=d u_{1} \cdots d u_{n}$. Because the absolute value of the last integral is less than $\|\mu\| \cdot z^{-n / 2}$, the proof of this lemma is complete.

TheOREM 3.3. Let $(B, H, m)$ be an abstract Wiener space and let $\left\{h_{1}, \ldots, h_{n}\right\}$ be as in Definition 2.3. Let $F: B \rightarrow \mathbb{C}$ be given by (3.1). Then for every $z \in \mathbb{C}_{+}$, the analytic Wiener integral $I^{\mathrm{aw}}(F ; z)$ of $F$ is expressed as follows:

$$
I^{\mathrm{aw}}(F ; z)=z^{n / 2} \int_{B} \exp \left\{\frac{(1-z)}{2} \sum_{j=1}^{n}\left[\left(h_{j}, x\right)^{\sim}\right]^{2}\right\} F(x) d m(x) .
$$

Proof. By Lemma 3.2, the right-hand side of (3.7) has a finite value. Now let us calculate the following Wiener integral:

$$
\begin{aligned}
\int_{B} \exp \left\{\frac{(1-z)}{2} \sum_{j=1}^{n}\left[\left(h_{j}, x\right)^{\sim}\right]^{2}\right\} F(x) d m(x) \\
=\left(\frac{1}{2 \pi}\right)^{n / 2} \int_{\mathbb{R}^{n}} \hat{\mu}(\vec{u}) \exp \left\{-\frac{z}{2}|\vec{u}|^{2}\right\} d \vec{u} \\
=\left(\frac{1}{2 \pi}\right)^{n / 2} \int_{\mathbb{R}^{n}}\left[\int_{\mathbb{R}^{n}} \exp \left\{i \sum_{j=1}^{n} v_{j} u_{j}\right\} \mu(d \vec{v})\right] \exp \left\{-\frac{z}{2}|\vec{u}|^{2}\right\} d \vec{u} \\
=\left(\frac{1}{2 \pi}\right)^{n / 2} \int_{\mathbb{R}^{n}}\left[\prod_{j=1}^{n}\left\{\int_{\mathbb{R}^{2}} \exp \left\{-\frac{z}{2} u_{j}^{2}+i v_{j} u_{j}\right\} d u_{j}\right\}\right] \mu(d \vec{v}) \\
=z^{-n / 2} \int_{\mathbb{R}^{n}} \exp \left\{-\frac{1}{2 z}|\vec{v}|^{2}\right\} \mu(d \vec{v}) .
\end{aligned}
$$

Here, the first equality comes from Theorem 2.4, the second equality comes from the definition of Fourier transform $\hat{\mu}$ of $\mu \in M\left(\mathbb{R}^{n}\right)$, the third equality follows from Fubini's theorem, and the last equality follows from the formula (2.10). From (3.2) and (3.8), we have the desired result (3.7). 
Now we express the analytic Feynman integral $I^{\text {af }}(F ; q)$ of $F$ as the limit of a sequence of Wiener integrals on the abstract Wiener space.

THEOREM 3.4. Let $(B, H, m)$ be an abstract Wiener space and let $\left\{h_{1}, \ldots, h_{n}\right\}$ be an orthonormal set of elements in H. Let $F: B \rightarrow \mathbb{C}$ be given by (3.1). If $\left\{z_{k}\right\}_{k=1}^{\infty}$ is a sequence of complex numbers from $\mathbb{C}_{+}$such that $z_{k}$ approaches $-i q$ through $\mathbb{C}_{+}$, where $q$ is $a$ nonzero real number and $i^{2}=-1$, then the analytic Feynman integral $I^{\text {af }}(F ; q)$ of $F$ is expressed as follows:

$$
I^{\mathrm{af}}(F ; q)=\lim _{k \rightarrow \infty}\left(z_{k}\right)^{n / 2} \int_{B} \exp \left\{\frac{1-z_{k}}{2} \sum_{j=1}^{n}\left[\left(h_{j}, x\right)^{\sim}\right]^{2}\right\} F(x) d m(x) .
$$

Proof. We can obtain from (3.8) that

$$
z_{k}^{n / 2} \int_{B} \exp \left\{\frac{\left(1-z_{k}\right)}{2} \sum_{j=1}^{n}\left[\left(h_{j}, x\right)^{\sim}\right]^{2}\right\} F(x) d m(x)=\int_{\mathbb{R}^{n}} \exp \left\{-\frac{1}{2 z_{k}}|\vec{v}|^{2}\right\} \mu(d \vec{v}) \text {. }
$$

Letting $k \rightarrow \infty$ in (3.10) and using the dominated convergence theorem, we have

$$
\begin{gathered}
\lim _{k \rightarrow \infty}\left(z_{k}\right)^{n / 2} \int_{B} \exp \left\{\frac{1-z_{k}}{2} \sum_{j=1}^{n}\left[\left(h_{j}, x\right)^{\sim}\right]^{2}\right\} F(x) d m(x) \\
=\lim _{k \rightarrow \infty} \int_{\mathbb{R}^{n}} \exp \left\{-\frac{1}{2 z_{k}}|\vec{v}|^{2}\right\} \mu(d \vec{v}) \\
=\int_{\mathbb{R}^{n}} \exp \left\{-\frac{i}{2 q}|\vec{v}|^{2}\right\} \mu(d \vec{v}) .
\end{gathered}
$$

From (3.2) and (3.11), (3.9) follows immediately.

Finally, we obtain a change of scale formula for Wiener integrals for $F: B \rightarrow \mathbb{C}$ which was given by (3.1).

THEOREM 3.5. Let $(B, H, m)$ be an abstract Wiener space. Let $\rho>0$ be given and let $\left\{h_{1}, \ldots, h_{n}\right\}$ be an orthonormal set of elements in $H$. Then for $F: B \rightarrow \mathbb{C}$ which was given by (3.1),

$$
\int_{B} F(\rho x) d m(x)=\rho^{-n} \int_{B} \exp \left\{\frac{\rho^{2}-1}{2 \rho^{2}} \sum_{j=1}^{n}\left[\left(h_{j}, x\right)^{\sim}\right]^{2}\right\} F(x) d m(x) .
$$

Proof. First, we know that for all real $z>0, I^{\mathrm{aw}}(F ; z)=\int_{B} F\left(z^{-1 / 2} x\right) d m(x)$ by Definition 2.2. Using Theorem 3.3 and taking $z=\rho^{-2}$ in the above equality, we have the desired result.

ACKNOWLEDGement. This paper was supported by BK-21 Project.

\section{REFERENCES}

[1] D. M. Chung, Scale-invariant measurability in abstract Wiener spaces, Pacific J. Math. 130 (1987), no. 1, 27-40. MR 88m:28009. Zbl 634.28007.

[2] G. Kallianpur and C. Bromley, Generalized Feynman integrals using analytic continuation in several complex variables, Stochastic Analysis and Applications, pp. 217267, Adv. Probab. Related Topics, vol. 7, Dekker, New York, 1984. MR 86m:60007. Zbl 554.60061. 
[3] Y. S. Kim, A change of scale formula for Wiener integrals of cylinder functions on abstract Wiener space, Int. J. Math. Math. Sci. 21 (1998), no. 1, 73-78. CMP 1486959. Zbl 891.28010.

Young Sik Kim: BK-21 Mathematical Science Division, Department of Mathematics, SeOUl NATiOnAL University, SEOUL, 151-742, Korea 


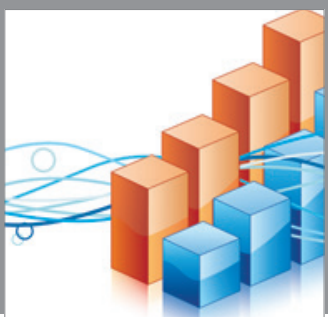

Advances in

Operations Research

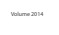

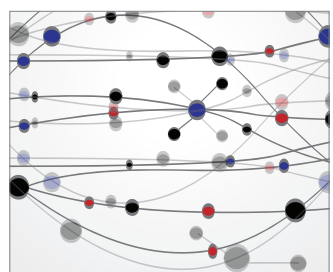

\section{The Scientific} World Journal
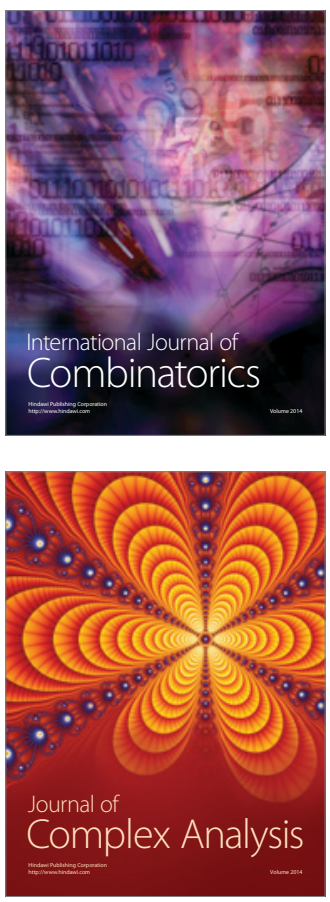

International Journal of

Mathematics and

Mathematical

Sciences
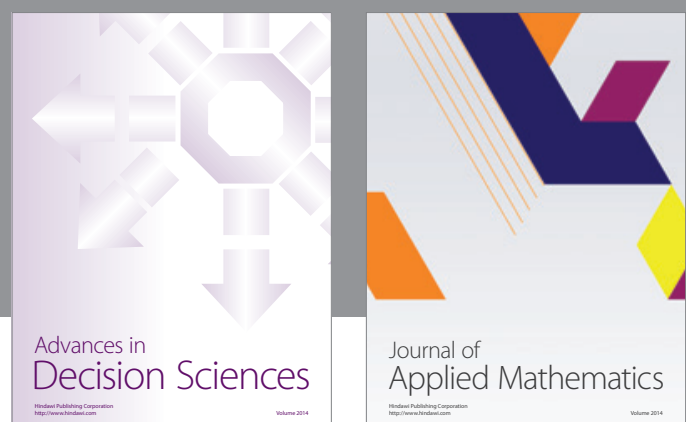

Journal of

Applied Mathematics
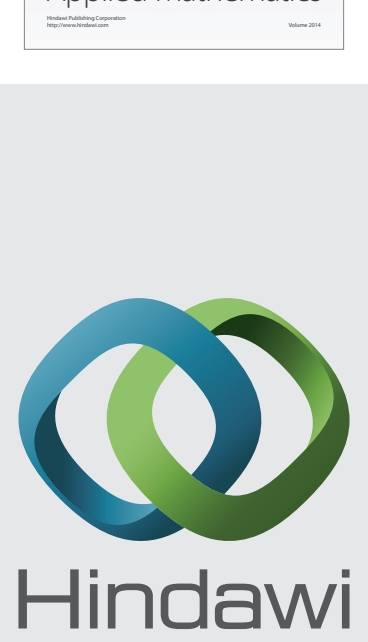

Submit your manuscripts at http://www.hindawi.com
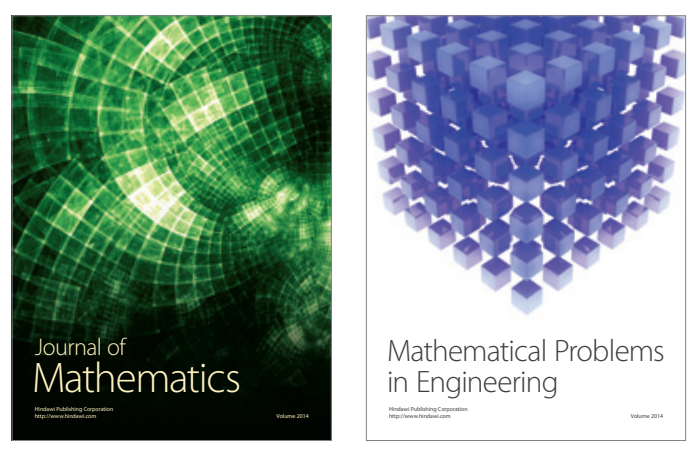

Mathematical Problems in Engineering
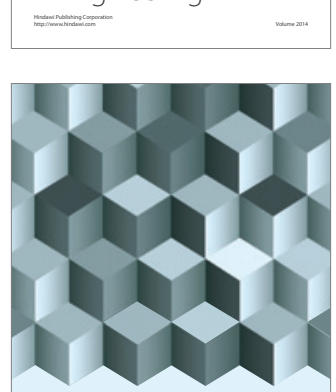

Journal of

Function Spaces
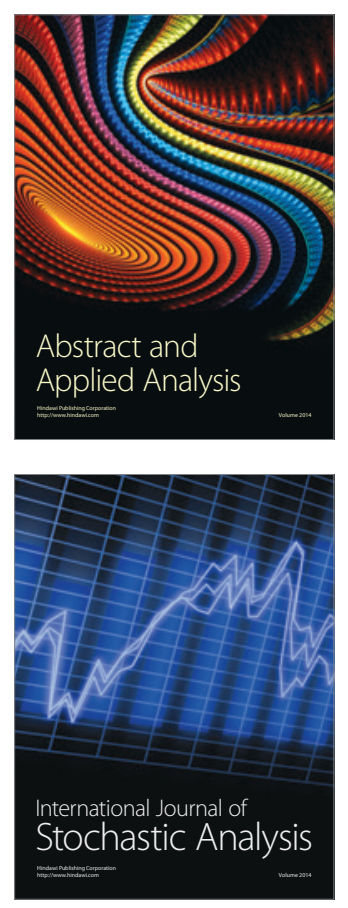

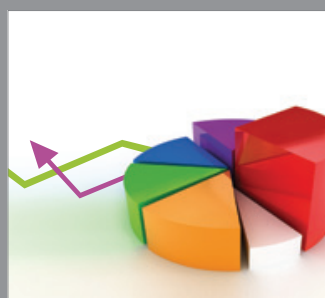

ournal of

Probability and Statistics

Promensencen
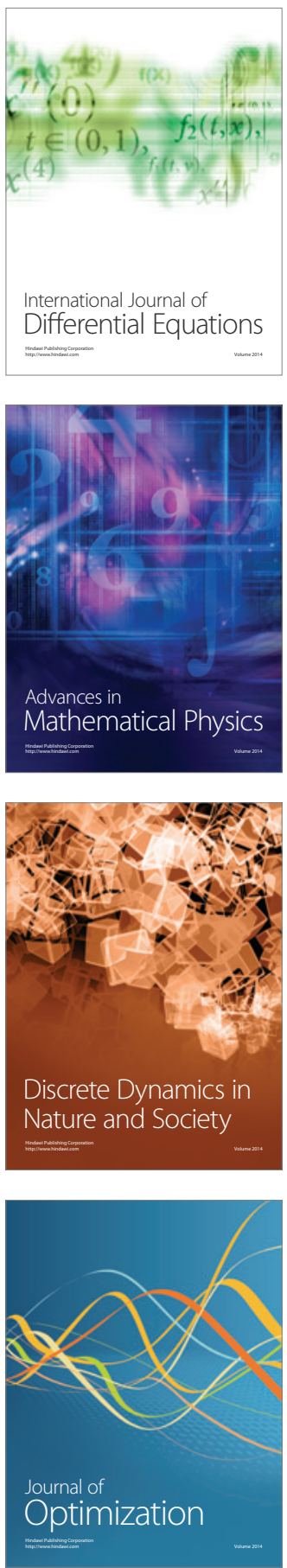